\title{
A General PDE-Framework for Registration of Contrast Enhanced Images
}

\author{
Mehran Ebrahimi and Anne L. Martel \\ Department of Medical Biophysics, University of Toronto \\ Imaging Research, Sunnybrook Health Sciences Centre \\ Toronto, Ontario, Canada \\ mehran.ebrahimi@sri.utoronto.ca, anne.martel@sri.utoronto.ca
}

\begin{abstract}
This paper presents a general PDE-framework for registration of contrast enhanced images. The approach directly applies the idea of separating the contrast enhancement term from the images in the regularization terms. In our formulation, we stay consistent with existing non-parametric image registration techniques, however, we carry an additional contrast enhancement term throughout. A mathematically rigorous approach is pursued which can exploit various forms of regularization. In this paper, our experiments are built based on diffusion regularization for both contrast enhancement and the deformation field.
\end{abstract}

\section{Introduction}

This paper provides a general mathematical framework to complement a series of algorithms [1 2 34 that support registration of contrast enhanced images.

Following Horn-Schunck's seminal work [5] on estimating the motion using optical flow equations, a number of approaches were proposed in the literature 6 6 34 which allowed varying illumination among the two images being registered. The approaches developed in 34] were very similar to Horn-Schunck's, except that they separated the illumination change as a linear intensity shift term. More recently, a similar idea has been applied to the registration of contrastenhanced medical images [21]. The common intuition behind all these methods is separating and regularizing the contrast enhancement term from the images in the regularization expression coupled with the motion. Numerically, these approaches propose constructing a very large system of equations directly based on the slightly modified optical flow equations. Iterative solvers (e.g., conjugate gradient method) are used to minimize the objective functional and estimate the deformation vectors. All of these methods used the diffusion penalty [7] function for the deformation field in practice and application of other regularization expressions is not readily available. In this paper, we present a general PDEframework for registration of contrast enhanced images. A goal of our work is to explain, complete, and extend the described set of methods.

To proceed, we need to rigorously introduce the required background material which will be employed in our formulation. For consistency, we adapt our notations from [7. In Section 2, we will introduce the mathematical formulation and 
problem set-up. In Section 3, we derive a PDE with a steady-state solution that corresponds to the solution of the described problem. The discretization and derivation of a numerical scheme for the PDE is followed in Section 4. Finally, we will present various computational experiments and concluding remarks in Sections 5 and 6 .

\section{Mathematical Formulation}

Consider the registration problem of a template image $T$ to a reference image $R$, where $T$ is a realization of $R$ deformed via a vector field $u$ and the contrast of a portion of this realization is changed via an extra additive term (image) $w$.

In this paper, the $d$-dimensional reference and template images are represented by mappings $R, T: \Omega \subset \mathbb{R}^{d} \rightarrow \mathbb{R}$ of compact support. The goal is to find a displacement vector field $u=\left(u_{1}, \ldots, u_{d}\right)$ and a compactly supported contrast enhancement image $w: \Omega \subset \mathbb{R}^{d} \rightarrow \mathbb{R}$ such that $T_{u}-w$ is similar to $R$, in which $T_{u}=T(x-u(x))$ is the deformed image and $T_{u}-w=T(x-u(x))-w(x)$ is the corrected-contrast deformed image. We formulate the registration of a contrasted-enhanced deformed image $T$ to a reference image $R$ as following.

Problem 1. Given two images $R$ and $T$, find a deformation $u$ and a contrast enhancement image $w$, that minimizes

$$
\mathcal{J}[u, w]:=\mathcal{D}[R, T ; u, w]+\mathcal{H}[u, w]
$$

in which $\mathcal{D}$ measures the similarity of $T_{u}-w$ and $R$, and $\mathcal{H}$ is a regularization expression on $[u, w]$. Here, assuming positive regularizing parameters $\alpha, \beta \in \mathbb{R}^{+}$ we express

$$
\mathcal{H}[u, w]:=\alpha \mathcal{P}[u]+\beta \mathcal{Q}[w] .
$$

Also, we use the sum of squares of intensity differences for the similarity measure

$$
\mathcal{D}[R, T ; u, w]:=\frac{1}{2}\left\|T_{u}-w-R\right\|_{L_{2}(\Omega)}^{2} .
$$

Hence, the objective is to minimize

$$
\mathcal{J}[u, w]:=\frac{1}{2}\left\|T_{u}-R-w\right\|_{L_{2}(\Omega)}^{2}+\alpha \mathcal{P}[u]+\beta \mathcal{Q}[w] .
$$

We shall present a mathematical formulation to solve Problem 1. Briefly speaking, we seek necessary conditions for optimality of $[u, w]$ by finding the Gâteaux derivatives of the components of $\mathcal{J}$ with respect to $[u, w]$. This shall provide us with the corresponding Euler-Lagrange equations that will be used to form a PDE which will be solved numerically.

Theorem 1. Let $d \in \mathbb{N}$, and $R, T$, and $w$ are $d$-dimensional real-valued images, i.e., functions from $\mathbb{R}^{d} \rightarrow \mathbb{R}, T \in C^{2}\left(\mathbb{R}^{d}\right), u: \mathbb{R}^{d} \rightarrow \mathbb{R}^{d}, v: \mathbb{R}^{d} \rightarrow \mathbb{R}^{d+1}$, $\Omega:=] 0, n\left[{ }^{d}\right.$. The Gâteaux derivative of $\mathcal{D}[R, T ; u, w]$ is given by

$$
d \mathcal{D}[R, T ; u, w ; v]=-\int_{\Omega}\langle\Phi(x, u(x), w(x)), v(x)\rangle_{\mathbb{R}^{d+1}} d x,
$$

in which $\Phi: \mathbb{R}^{d} \times \mathbb{R}^{d} \times \mathbb{R} \rightarrow \mathbb{R}^{d+1}$, 


$$
\Phi(x, u(x), w(x))=\left[T_{u}(x)-R(x)-w(x)\right]\left(\nabla T_{u}(x), 1\right) .
$$

[see the proof in Appendix 1., Cf. [7] pp. 80.]

Here, we focus on the special case where $\mathcal{P}, \mathcal{Q}$ are diffusion regularization expressions 7895431011 .

Theorem 2. Assume $\mathcal{P}$ and $\mathcal{Q}$ are diffusion regularization expressions and the functionals $\mathcal{P}^{e}$ and $\mathcal{Q}^{e}$ are respectively extensions of $\mathcal{P}$ and $\mathcal{Q}$, i.e.,

$$
\begin{aligned}
& \mathcal{P}^{e}[(u, w)]:=\mathcal{P}[u]:=\frac{1}{2} \sum_{j=1}^{d} \int_{\Omega}\left\langle\nabla u_{j}, \nabla u_{j}\right\rangle d x, \\
& \mathcal{Q}^{e}[(u, w)]:=\mathcal{Q}[w]:=\frac{1}{2} \int_{\Omega}\langle\nabla w, \nabla w\rangle d x .
\end{aligned}
$$

Also, assume that Neumann boundary conditions are imposed, i.e.,

$$
\langle\nabla w(x), \vec{n}(x)\rangle_{\mathbb{R}^{d}}=\left\langle\nabla u_{j}(x), \vec{n}(x)\right\rangle_{\mathbb{R}^{d}}=0 \quad \text { for } \quad x \in \partial \Omega \text { and } \quad j=1, \ldots, d,
$$

in which $\vec{n}$ denotes the outer normal unit vector of $\partial \Omega$ (boundary of $\Omega$ ). The Gâteaux derivative of $\mathcal{P}^{e}[(u, w) ; v]$ and $\mathcal{Q}^{e}[(u, w) ; v]$ are respectively

$$
\begin{aligned}
& d \mathcal{P}^{e}[(u, w) ; v]=-\int_{\Omega}\langle\mathcal{A}[u](x), v(x)\rangle_{\mathbb{R}^{d+1}} d x, \\
& d \mathcal{Q}^{e}[(u, w) ; v]=-\int_{\Omega}\langle\mathcal{B}[w](x), v(x)\rangle_{\mathbb{R}^{d+1}} d x
\end{aligned}
$$

where,

$$
\begin{aligned}
& \mathcal{A}[u](x)=\left(\Delta u_{1}(x), \ldots, \Delta u_{d}(x), 0\right)=(\Delta u(x), 0), \\
& \mathcal{B}[w](x)=(\underbrace{0, \ldots, 0}_{d_{\text {times }}}, \Delta w(x))=\left(0_{\mathbb{R}^{d}}, \Delta w(x)\right) .
\end{aligned}
$$

Proof. The result yields applying the Green's formula similar to [7] pp. 138.

Theorem 3. The Euler-Lagrange equations corresponding to $\mathcal{J}=\mathcal{D}+\alpha \mathcal{P}+\beta \mathcal{Q}$, where $\mathcal{D}$ is defined by Equation (2) and $\mathcal{P}, \mathcal{Q}$ are defined by Equations (34) respectively are

$$
\Phi(x, u(x), w(x))+\alpha \mathcal{A}[u](x)+\beta \mathcal{B}[w](x)=0, \quad x \in \Omega,
$$

with Neumann boundary conditions. These can also be written as

$$
\begin{array}{cc}
{\left[T_{u}(x)-R(x)-w(x)\right] \nabla T_{u}(x)+\alpha \Delta u(x)=0_{\mathbb{R}^{d}}} & x \in \Omega, \\
{\left[T_{u}(x)-R(x)-w(x)\right]+\beta \Delta w(x)=0} & x \in \Omega, \\
\langle\nabla w(x), \vec{n}(x)\rangle_{\mathbb{R}^{d}}=\left\langle\nabla u_{l}(x), \vec{n}(x)\right\rangle_{\mathbb{R}^{d}}=0, l=1, \ldots, d, x \in \partial \Omega .
\end{array}
$$

Proof. The result yields using substitution (Cf. 7] pp. 138.) . 


\section{A Corresponding PDE}

There exist various ways to solve Equation (5). A possibility that we pursue here is to formulate the solution as the steady-state solution of a corresponding PDE similar to [7]. We propose

$\partial_{t}(u(x, t), s w(x, t))=\Phi(x, u(x, t), w(x, t))+\alpha \mathcal{A}[u](x)+\beta \mathcal{B}[w](x) \quad x \in \Omega, t \geq 0$, where $s$ is a scale factor. Assuming $\Phi=(f, g)$ the PDE can be written as

$$
\begin{aligned}
\partial_{t} u(x, t) & =f(x, u(x, t), w(x, t))+\alpha \Delta u(x, t), \quad x \in \Omega, \quad t \geq 0, \\
s \partial_{t} w(x, t) & =g(x, u(x, t), w(x, t))+\beta \Delta w(x, t), \quad x \in \Omega, \quad t \geq 0, \\
f(x, u, w) & :=\left[T_{u}(x)-R(x)-w(x)\right] \nabla T_{u}(x), \\
g(x, u, w) & :=\left[T_{u}(x)-R(x)-w(x)\right] .
\end{aligned}
$$

\section{Discretization and Numerical Scheme}

To numerically solve the derived PDE in Equations (6]7), we evaluate expressions at discrete time variable $\left\{t_{k+1}\right\}$

$$
\begin{gathered}
\partial_{t} u\left(x, t_{k+1}\right)=f\left(x, u\left(x, t_{k}\right), w\left(x, t_{k}\right)\right)+\alpha \Delta u\left(x, t_{k+1}\right), \quad x \in \Omega, \\
s \partial_{t} w\left(x, t_{k+1}\right)=g\left(x, u\left(x, t_{k+1}\right), w\left(x, t_{k+1}\right)\right)+\beta \Delta w\left(x, t_{k+1}\right), \quad x \in \Omega .
\end{gathered}
$$

Notice that due to the nonlinearity of $f$ with respect to $u, f$ is evaluated at $t_{k}$ instead of $t_{k+1}$ in Equation (8) [cf. [7] pp. 80] which translates to applying a fixedpoint iteration scheme. However, $g$ is linear with respect to $w$ and $t_{k+1}$ is used consistently in Equation (9). Using a spatial discretization $X$ of $\Omega$ that includes $n^{d}$ voxels (pixels) corresponding to a unit space step in every dimension due to the definition of $\Omega:=] 0, n\left[{ }^{d}\right.$, and a time step of $\tau_{1}$, we define for $j=1, \ldots, d$, and $k \in \mathbb{Z}^{*}$ (the set of non-negative integers)

$$
\begin{aligned}
& U_{j}^{k}(X):=u_{j}\left(X, \tau_{1} k\right):=\operatorname{Discretized}\left(u_{j}\left(x, t_{k}\right)\right), \\
& W^{k}(X):=w\left(X, \tau_{1} k\right):=\operatorname{Discretized}\left(w\left(x, t_{k}\right)\right) .
\end{aligned}
$$

Furthermore, $\quad A U_{j}^{k}:=\Delta u_{j}\left(X, \tau_{1} k\right):=\operatorname{Discretized}\left(\Delta u_{j}\left(x, t_{k}\right)\right)$,

$$
A W^{k}:=\Delta w\left(X, \tau_{1} k\right):=\operatorname{Discretized}\left(\Delta w\left(x, t_{k}\right)\right),
$$

in which $A \in \mathbb{R}^{n^{d} \times n^{d}}$ is defined such that 


$$
A U_{j}^{k} \approx \sum_{l=1}^{d} \partial_{x_{l}, x_{l}} u_{j}\left(X, \tau_{1} k\right) \text { and } A W^{k} \approx \sum_{l=1}^{d} \partial_{x_{l}, x_{l}} w\left(X, \tau_{1} k\right)
$$

[See Appendix 2 for the precise definition of $A$.]

Substituting the discretization in the PDEs of Equations (8,9) leads that for $j=1, \ldots, d, k \in \mathbb{Z}^{*}$

$$
\begin{aligned}
& \frac{U_{j}^{k+1}-U_{j}^{k}}{\tau_{1}}=\left(T\left(X-U^{k}(X)\right)-R(X)-W^{k}(X)\right) \partial_{j} T\left(X-U^{k}(X)\right)+\alpha A U_{j}^{k+1}, \\
& s \frac{W^{k+1}-W^{k}}{\tau_{1}}=\left(T\left(X-U^{k+1}(X)\right)-R(X)-W^{k+1}(X)\right)+\beta A W^{k+1} .
\end{aligned}
$$

Defining $\tau_{2}:=\tau_{1} / s$ gives

$$
\begin{aligned}
& \left(I-\tau_{1} \alpha A\right) U_{j}^{k+1}=U_{j}^{k}+\tau_{1}\left(T\left(X-U^{k}(X)\right)-R(X)-W^{k}(X)\right) \partial_{j} T\left(X-U^{k}(X)\right), \\
& \left(\left(1+\tau_{2}\right) I-\tau_{2} \beta A\right) W^{k+1}=W^{k}+\tau_{2}\left(T\left(X-U^{k+1}(X)\right)-R(X)\right),
\end{aligned}
$$

where $I \in \mathbb{R}^{n^{d} \times n^{d}}$ is the identity matrix. This yields

$$
\begin{aligned}
& U_{j}^{k+1}=\left(I-\tau_{1} \alpha A\right)^{-1}\left[U_{j}^{k}+\tau_{1}\left(T\left(X-U^{k}(X)\right)-R(X)-W^{k}(X)\right) \partial_{j} T\left(X-U^{k}(X)\right)\right] \\
& W^{k+1}=\left(\left(1+\tau_{2}\right) I-\tau_{2} \beta A\right)^{-1}\left[W^{k}+\tau_{2}\left(T\left(X-U^{k+1}(X)\right)-R(X)\right)\right] .
\end{aligned}
$$

Finally, we use the initialization vectors $W^{0}=U_{j}^{0}=0_{\mathbb{R}^{n^{d}}}, \quad j=1, \ldots, d$.

\section{Computational Experiments}

To better understand the introduced scheme, we start from two 2-dimensional 8bit, $256 \times 256$ images shown in Fig. 1(a1-a2) i.e., $d=2, n=256$. The intensities of images have also been mapped to $[0,1]$. The reference image is generated by deforming the template using a finite element model (FEM), where the magnitude of the deformation is shown in Fig. $1 \mathrm{~b}(1)$, followed by subtracting a contrast enhancement term shown in Fig. $1 b(3)$ from the deformed template image. The contrast enhancement term is generated as a combination of physiological enhancement term taken from a patient study and an artificial gradient term.

We apply the scheme derived in Equation (10) to evaluate the deformation and the contrast enhancement terms given only the reference and template image. In all of these experiments, we fix the number of iterations to 50 , choose $\alpha=0.1$, $\tau_{1}=10^{3}, \tau_{2}=10^{10}$ and vary the parameter $\beta$. The result are shown in rows (c-f) of Figure 1. The first four columns in each row respectively corresponds to the 


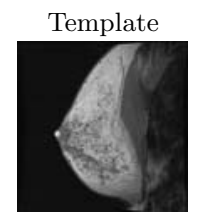

(a1) $T$

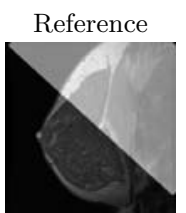

(a2) $R$
Difference

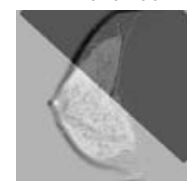

(a3) $T-R$

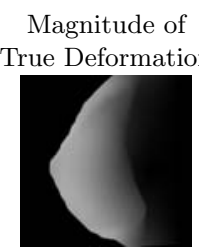

(b1) $\left\|u^{*}\right\|$

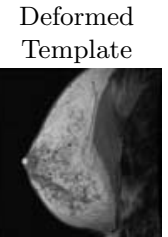

(b2) $T_{u^{*}}$
True Contrast

Ehancement

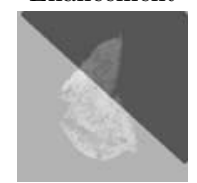

(b3) $T_{u^{*}}-R$

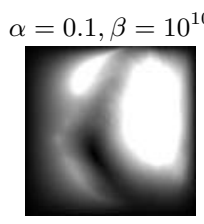

(c1) $\|u\|$

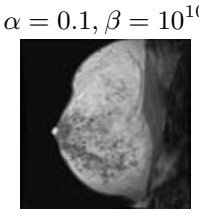

(c2) $T_{u}$

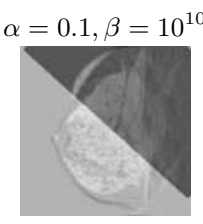

(c3) $T_{u}-R$

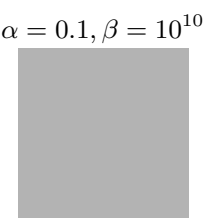

(c4) $w$

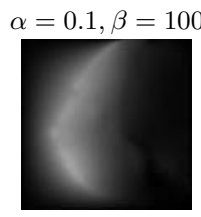

(d1) $\|u\|$

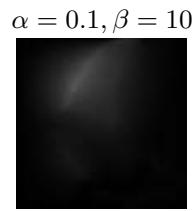

(e1) $\|u\|$

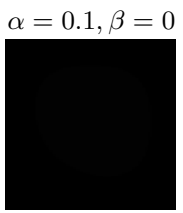

(f1) $\|u\|$

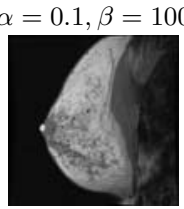

(d2) $T_{u}$

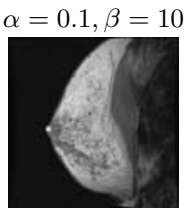

(e2) $T_{u}$

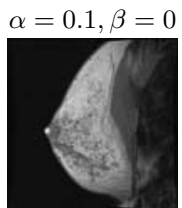

(f2) $T_{u}$

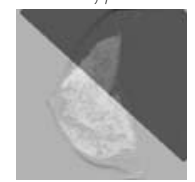

(d3) $T_{u}-R$

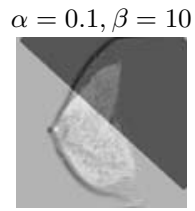

(e3) $T_{u}-R$

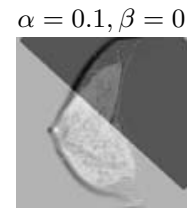

(f3) $T_{u}-R$ (d4) $w$
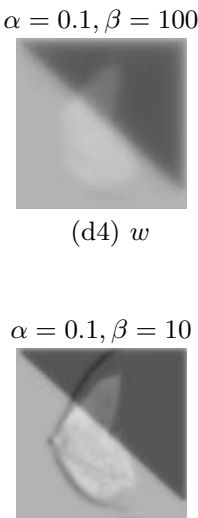

(e4) $w$

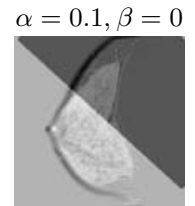

(f4) $w$

Fig. 1. Computational experiments on simulated data 
magnitude of the corresponding deformation shown in a [0-15] gray-level map, the deformed template, the difference between the deformed template and the reference, and the computed contrast enhancement. The employed parameter $\beta$ for each row is indicated above the Figures.

The case $\beta=10^{10}$ shown in row (c) corresponds to a diffusion-based registration ignoring the contrast term, while the case $\beta=0$ in row (f) corresponds to the case that contrast term is not penalized and can vary arbitrarily. It can be observed that among the selected parameters $\beta$, the choice of $\beta=100$ shown in row (d) gives the most reasonable estimation of the motion and the contrast enhancement term.

\section{Concluding Remarks}

We offered a mathematical framework for registration of contrast enhanced images. Our approach directly applies the idea of separating the contrast enhancement term from the images in regularization $344_{12}$. We applied diffusion regularizations for both $u$ and $w$, where the idea of Adaptive Operator Splitting (AOS) can be simply used to split the operator $A$ of Equation (10) to present a scheme of linear complexity similar to [7. Finally, the choice of regularizer $\mathcal{H}[u, w]$ in Equation (1) may be replaced with other suitable expressions.

Acknowledgments. This research was supported in part by the Natural Sciences and Engineering Research Council of Canada (NSERC) in the form of a Postdoctoral Fellowship for Mehran Ebrahimi. This work was also supported by the Terry Fox Foundation for Cancer Research. The authors thank Dr. Ellen Warner (Sunnybrook Health Sciences Centre) and Dr. Kristy Brock (Princess Margaret Hospital) for providing the MR data and FEM simulations.

\section{References}

1. Martel, A.L., Plewes, D.B., Froh, M.S., Brock, K.K., Barber, D.C.: Evaluating an optical-flow-based registration algorithm for contrast-enhanced magnetic resonance imaging of the breast. Physics in Medicine and Biology 52(13), 3803-3816 (2007)

2. Barber, D.C., Hose, D.R.: Automatic segmentation of medical images using image registration: diagnostic and simulation applications. Journal of medical engineering and technology 29(2), 53-63 (2005)

3. Gennert, M.A., Negahdaripour, S.: Relaxing the brightness constancy assumption in computing optical flow. 62 Computers, Control and Information Theory AIMEMO975; ADA1874379, MIT (June 1987)

4. Negahdaripour, S., Yu, C.H.: A generalized brightness change model for computing optical flow. In: 1993 IEEE 4th International Conference on Computer Vision, pp. 2-11. IEEE Computer Society Press, Los Alamitos (1993)

5. Horn, B.K.P., Schunck, B.G.: Determining optical flow. Artificial Intelligence 17(13; special volume), 185-203 (1981) 
6. Weickert, J., Bruhn, A., Brox, T., Papenberg, N.: A survey of variational optic flow methods for small displacement. In: Mathematical Models for Registration and Applications to Medial Imaging, Springer, Heidelberg (2005)

7. Modersitzki, J.: Numerical methods for image registration. Oxford University Press, Oxford (2004)

8. Fischer, B., Modersitzki, J.: Ill-posed medicine-an introduction to image registration. Inverse Problems 24(3), 16 (2008); 034008

9. Fischer, B., Modersitzki, J.: Fast image registration: a variational approach. In: Applied Numerical Analysis and Computational Mathematics, 2004 (NACoM-2003), Cambridge, UK, Germany, vol. 1, pp. A69-A74. Wiley-VCH, Chichester (2004)

10. Thirion, J.P.: Fast non-rigid matching of 3d medical images. In: Medical Robotics and Computer Aided Surgery, Baltimore, pp. 47-54 (1995)

11. Thirion, J.P.: Image matching as a diffusion process: an analogy with Maxwell's demons. Medical Image Analysis 2(3), 243-260 (1998)

12. Ebrahimi, M., Martel, A.L.: Image registration under varying illumination: Hyperdemons algorithm. (preprint, 2009)

\section{Appendix 1: Proof of Theorem 1}

Proof. Define $p(x):=\left(v_{1}(x), \ldots, v_{d}(x)\right)$ and $q(x):=v_{d+1}(x)$, i.e., split $v(x)$ to $v(x)=(p(x), q(x))$. Using the Taylor expansion of $T_{u+h p}(x)$ with respect to $h$ at the point $x-u(x)$,

$$
\left.T_{u+h p}(x)=T(x-u(x)-h p(x))\right)=T_{u}(x)-h\left\langle\nabla T_{u}(x), p(x)\right\rangle_{\mathbb{R}^{d}}+\mathcal{O}\left(h^{2}\right) .
$$

Hence, (Cf. 7] pp. 81.)

$$
\begin{array}{rl}
d & \mathcal{D}[R, T ; u, w ; v]=\lim _{h \rightarrow 0} \frac{1}{h}(\mathcal{D}[R, T ;(u, w)+h v]-\mathcal{D}[R, T ; u, w]) \\
= & \lim _{h \rightarrow 0} \frac{1}{h}(\mathcal{D}[R, T ;(u+h p, w+h q)]-\mathcal{D}[R, T ; u, w]) \\
= & \lim _{h \rightarrow 0} \frac{1}{2 h} \int_{\Omega}\left(T_{u+h p}(x)-R(x)-(w(x)+h q(x))\right)^{2} \\
- & \left(T_{u}(x)-R(x)-w(x)\right)^{2} d x \\
= & \lim _{h \rightarrow 0} \frac{1}{2 h} \int_{\Omega}\left(T_{u}(x)-h\left\langle\nabla T_{u}(x), p(x)\right\rangle_{\mathbb{R}^{d}}+\mathcal{O}\left(h^{2}\right)-R(x)-w(x)-h q(x)\right)^{2} \\
- & \left(T_{u}(x)-R(x)-w(x)\right)^{2} d x \\
= & \lim _{h \rightarrow 0} \frac{1}{2 h} \int_{\Omega} 2\left[T_{u}(x)-R(x)-w(x)\right]\left(-h\left\langle\nabla T_{u}(x), p(x)\right\rangle_{\mathbb{R}^{d}}-h q(x)+\mathcal{O}\left(h^{2}\right)\right) d x \\
= & \int_{\Omega}-\left[T_{u}(x)-R(x)-w(x)\right]\left[\left\langle\nabla T_{u}(x), p(x)\right\rangle_{\mathbb{R}^{d}}+\langle 1, q(x)\rangle_{\mathbb{R}}\right] d x \\
= & \int_{\Omega}\left\langle-\left[T_{u}(x)-R(x)-w(x)\right]\left(\nabla T_{u}(x), 1\right), v(x)\right\rangle_{\mathbb{R}^{d+1}} d x .
\end{array}
$$




\section{Appendix 2: Definition of $A$}

$A \in \mathbb{R}^{n^{d} \times n^{d}}$ is defined as $A:=\sum_{l=1}^{d} A_{l}$ where $A_{l}=\underbrace{I \otimes \cdots \otimes I}_{l-1 \text { times }} \otimes B \otimes \underbrace{I \otimes \cdots \otimes I}_{d-l \text { times }}$, in which $I \in \mathbb{R}^{n \times n}$ is identity matrix and $\otimes$ denotes the Kronecker product of matrices. The $l^{\text {th }}$ factor $B \in \mathbb{R}^{n \times n}$ is an approximation of the second order derivative in only one spatial direction. More precisely, it can be defined as the tridiagonal matrix

$$
B=\left(\begin{array}{ccccc}
-2 & 1 & 0 & \ldots & 0 \\
1 & -2 & 1 & \ldots & 0 \\
\vdots & \ddots & \ddots & \ddots & \vdots \\
0 & \ldots & 1 & -2 & 1 \\
0 & \ldots & \ldots & 1 & -2
\end{array}\right)
$$

